\title{
Ehebruch, Zanksucht, mangelhafte Haushaltsführung ... Scheidungsgründe im Wandel der Zeit
}

From the Ehegesetz of 1938 to its reform in 1999, adultery, refusal of reproduction and other matrimonial offences counted as grounds for divorce. With the absolute grounds for divorce, i.e. adultery and the refusal of reproduction, there was no further test on their destructive effects, whereas with the remaining ones, which were known as relative grounds, there had to be a test on whether the offence in question had indeed harmed the marital union.

Following the reform of 1999 (Eherechtsänderungsgesetz) only severe matrimonial offences remain as relative grounds for divorce. A matrimonial offence may be any given behaviour of a spouse which undermines the foundation of their marriage. Thus, not just unethical behaviour such as insults or physical assaults but also too close (though not sexual) contact with a person of the opposite sex may under certain circumstances constitute a ground for divorce.

In the past few years the claim of 'unloving behaviour', which may refer to a great variety of practices, has increasingly been brought forward in courts. For the judges it is getting more and more difficult to establish which of those should be regarded as a severe offence.

Vorausschickend wird angemerkt, dass der Vortrag nur die Zeit vom Ehegesetz 1938 bis heute umfasst. Auch davor gab es natürlich schon die Möglichkeit einer Ehescheidung - dieses Thema können aber Rechtshistoriker sicher besser abhandeln!

\section{Von absoluten und relativen} Scheidungsgründen

oder die Entwicklung der Ehescheidungsgründe vom Ehegesetz 1938 bis zum Eherechtsänderungsgesetz 1999

Das Ehegesetz 1938 regelte die Scheidung aus Verschulden wie folgt:

\section{„§ 47 Ehebruch}

1)Ein Ehegatte kann Scheidung begehren, wenn der andere die Ehe gebrochen hat.

2) Er hat kein Recht auf Scheidung, wenn er dem Ehebruch zugestimmt hat oder ihn durch sein Verhalten absichtlich ermöglicht oder erleichtert hat.

$\S 48$ Verweigerung der Fortpflanzung

Ein Ehegatte kann Scheidung begehren, wenn der andere sich ohne triftigen Grund beharrlich weigert, Nachkommenschaft zu erzeugen oder zu empfangen, oder wenn er rechtswidrig Mittel zur Verhinderung der Geburt anwendet oder anwenden lässt.

\section{$\S 49$ andere Eheverfehlungen}

Ein Ehegatte kann Scheidung begehren, wenn der andere durch eine sonstige Eheverfehlung oder durch ehrloses oder unsittliches Verhalten 
die Ehe schuldhaft so tief zerrüttet hat, dass die Widerherstellung einer ihrem Wesen entsprechenden Lebensgemeinschaft nicht erwartet werden kann.

Wer selbst eine Verfehlung begangen hat, kann die Scheidung nicht begehren, wenn nach der Art seiner Verfehlung insbesondere wegen des Zusammenhangs der Verfehlung des anderen Ehegatten mit seinem eigenen Verschulden sein Scheidungsbegehren bei richtiger Würdigung des Wesens der Ehe sittlich nicht gerechtfertigt ist."

Die besonderen Scheidungstatbestände des Ehebruches und der Verweigerung der Fortpflanzung wurden von der Rechtsprechung als absolute Scheidungsgründe verstanden, deren ehezerstörende Wirkung nicht geprüft werden durfte. Auch der bei der gänzlich zerrütteten Ehe oder nach Aufhebung der ehelichen Gemeinschaft begangene Ehebruch rechtfertigt die Scheidung aus dem Verschulden des Ehebruches. ${ }^{1}$

$\S 49$ verlangte hingegen, dass durch die Eheverfehlung eine Zerrüttung eintritt, die Eheverfehlung ist also relativ, weil ihre Geltendmachung als Scheidungsgrund voraussetzt, dass die Verfehlung die eheliche Gemeinschaft beeinträchtigt. Eine unheilbare Zerrüttung einer Ehe ist dann anzunehmen, wenn die geistige seelische und körperliche Gemeinschaft zwischen den Ehegatten und damit die Grundlage der Ehe objektiv und wenigstens bei einem der Ehegatten auch subjektiv zu bestehen aufgehört hat. ${ }^{2}$

Auch dann, wenn ein Gatte den anderen verlassen oder den Willen zur Ehe verloren hat, der Verlassene aber immer noch vergeblich auf die äußere und innere Rückkehr des anderen hofft und innerlich an der ehelichen Bindung festhält,

${ }^{1}$ OLG Wien 1. 9. 1980, 14 R 126/80.

${ }^{2}$ OGH 30. 4. 1980, 1 Ob 549/80. die der andere abgestreift hat, kann und wird auch die Ehe meist objektiv zerrüttet sein. ${ }^{3}$

Nach dem Eherechtsänderungsgesetz 1999 BGBl 1999/125 enthält nun der $\S 49$ EheG eine Generalklausel für alle auf Verschulden beruhende Scheidungsgründe und verlangt außer der schuldhaften schweren Eheverfehlung während der Ehe eine tiefgreifende Zerrüttung der Ehe, somit sind alle Scheidungsgründe nun als relative Scheidungsgründe einzustufen.

\section{„Scheidung wegen Verschuldens (Eheverfeh- lungen)}

Ein Ehegatte kann Scheidung begehren, wenn der andere durch eine schwere Eheverfehlung oder durch ehrloses oder unsittliches Verhalten die Ehe schuldhaft so tief zerrüttet hat, dass die Widerherstellung einer ihrem Wesen entsprechenden Lebensgemeinschaft nicht erwartet werden kann. Eine schwere Eheverfehlung liegt insbesondere dann vor, wenn ein Ehegatte die Ehe gebrochen hat oder dem anderen körperliche Gewalt oder schweres seelisches Leid zugefügt hat.

Wer selbst eine Verfehlung begangen hat, kann die Scheidung nicht begehren, wenn nach der Art seiner Verfehlung insbesondere wegen des Zusammenhangs der Verfehlung des anderen Ehegatten mit seinem eigenen Verschulden sein Scheidungsbegehren bei richtiger Würdigung des Wesens der Ehe sittlich nicht gerechtfertigt ist."

Das LG Salzburg fasste in seiner Entscheidung vom 15. März. 2000 zusammen: ${ }^{4}$ Eheverfehlungen sind Verletzungen der gesetzlichen Pflichten, wie sie sich aus den persönlichen Rechtswirkungen der Ehe ergeben und richten sich unmittelbar gegen den Partner. Aber auch der Begriff der Ehe in $\S 44$ ABGB ist auslegungsbedürftig:

\footnotetext{
${ }^{3}$ OLG Wien 14.4.1980, 14 R 43/80.

${ }^{4}$ LG Salzburg 15. 3.2000, 21 R 28/00i.
} 
Die Familien-Verhältnisse werden durch den Ehevertrag gegründet. In dem Ehevertrage erklären zwei Personen verschiedenen Geschlechts gesetzmäßig ihren Willen, in unzertrennlicher Gemeinschaft $z u$ leben, Kinder zu zeugen, sie zu erziehen und sich gegenseitig Beistand zu leisten.

Dazu kommt auch noch das sog. unsittliche Verhalten:

Ein unsittliches Verhalten kann auch dann einen Scheidungsgrund bilden, wenn es keine Ehepflicht verletzt. Unsittlich ist ein Verhalten, das den allgemeinen sittlichen Anschauungen widerspricht. Es wird zu einer schweren Eheverfehlung dann, wenn es die Grundlage der Ehe untergräbt. Vereinzelten Unschicklichkeiten kommt diese Eignung nicht zu. ${ }^{5}$

Das alles zeigt: zur Beurteilung der Frage, ob eine schwere Eheverfehlung vorliegt, muss das Gericht das Gesamtverhalten der Ehegatten während der Ehe prüfen! Eine schwierige Aufgabe für den/die Scheidungsrichter/in, denn nirgends sonst wird so emotional "die Wahrheit" erzählt. Die Beweiswürdigung bei Aussage gegen Aussage muss nicht immer gelingen. Letztlich gewinnt der „überzeugendere“ Ehegatte, dies hat die Richterschaft dazu bewogen wiederholt die gänzliche Abschaffung des Verschuldensprinzip zu fordern, denn die Rosenkriege werden durch den Druck das Verschulden des anderen beweisen $\mathrm{zu}$ müssen nur noch schlimmer....

Zur Vorbereitung dieses Vortrages wurden viele Entscheidungen durchforstet, auf der Suche nach Entscheidungen, die heute wohl nicht mehr so ergehen würden oder nach Entscheidungen, die eine gewisse Entwicklung des Scheidungsrechtes zeigen. Die Formulierungen der Gerichte verraten dabei oft mehr von der dahinterstehenden Weltanschauung als der Inhalt der Entscheidung selbst - darum werden im weiteren Text lauter Originalzitate folgen!

${ }^{5}$ OGH 11. 3. 1959, 5 Ob 120/59.
Der erste Band der EFSlg. umfasst die Jahre 1945 bis 1964, seither wird jährlich ein Band herausgegeben. Gesellschaftspolitische Tendenzen finden ihren Niederschlag in der scheidungsrechtlichen Judikatur genauso wie Veränderungen in der Arbeitswelt, Veränderungen im Umgang zwischen Mann und Frau usw.. Der Stoff für weitere Bände rund um gescheiterte Beziehungen und deren rechtliche Konsequenzen wird nicht ausgehen ...

\section{Ehebruch}

Wie bereits ausgeführt hielt der OGH bereits am 14. September. 1949 fest:

„,Der Scheidungsgrund des $\S 47$ EheG hat keine ehezerrüttende Wirkung zur Voraussetzung. Auch der nach Aufhebung der ehelichen Gemeinschaft begangene Ehebruch rechtfertigt die Scheidung aus dem Verschulden des Ehebrechers." ${ }^{6}$

Das Wort „Ehebrecher" würde der OGH heute wohl etwas umformulieren....

Gar nicht so lange her sondern aus 1950:

„Wenn sich eine Ehefrau unter dem Druck von Drohungen einem Geschlechtsverkehr mit einem Offizier der Besatzungsmacht hingibt, ist darin keine Eheverfehlungen im Sinne der $\S 47$ oder 49 EheG zu erblicken. ${ }^{\text {7 }}$

Oder aus 1964:

„,Ein, wenn auch grob fahrlässiger Irrtum über das Bestehen des Ehebandes (Tod des Ehegatten anlässlich der Kriegsereignisse) macht die Geltendmachung des Ehebruches als Scheidungsgrund unzulässig. " 8

Interessant formuliert, denn vielleicht wollte die Ehefrau gar nichts verhindern?

\footnotetext{
${ }^{6}$ OGH 14. 9. 1949, 3 Ob 230/49.

${ }^{7}$ OGH 20. 9. 1950, 3 Ob 314/50.

${ }^{8}$ OGH 10. 2. 1964, 1 Ob 2/64.
} 
„Ein von der Ehefrau wiederholt ohne Gegenwehr geduldeter Geschlechtsverkehr mit einem anderen Mann ist eine schwere Eheverfehlung, auch wenn er von ihr nicht gerade gesucht worden aber doch zu verhindern gewesen wäre." ${ }^{\prime 9}$

„Besuche bei Prostituierten bilden, ohne Kenntnis weiterer Einzelheiten, mögen sie auch vom anderen Ehepartner widerstrebend hingenommen worden sein, schwere Verstöße gegen die eheliche Treue, zu der die Eheleute nicht bloß im körperlichen sondern auch im geistig - seelischen Bereich verpflichtet sind. . ${ }^{10}$

Als Ehebruch iSd $\S 47$ EheG wurde nur der Vollzug des Beischlafes gesehen während die schwere Eheverfehlung bereits alle "Vorbereitungshandlungen zum Beischlaf" umfasst.

Der Beweis eines Beischlafes gelang nicht immer:

„Da dem häufigen Beisammensein des Beklagten mit einer anderen Frau ebenso wenig, wie seinem mit ihr verbrachten Urlaub im Sommer trotz der durch die äußeren Umstände bedingten gemeinsamen Übernachtung (zusammen mit der Tochter des Klägers) in einem Zimmer oder der gemeinsamen Reise nach Italien im gleichen Sommer und den späteren Ausflügen im Winter ein erotisches Motiv zugrunde lag und es dabei zu keinerlei Zärtlichkeiten oder gar geschlechtlichen Beziehungen kam, kann eine Verletzung seiner Treuepflicht gegenüber der Klägerin nicht erblickt werden. ${ }^{11}$

Ein Dauerbrenner der Scheidungsjudikatur ist der Umgang eines Ehegatten mit einer Person des anderen Geschlechts - nach wie vor gültig eine Entscheidung:

„Ein freundschaftlich vertrauter aber harmloser Verkehr mit einer Person des anderen Geschlechts stellt keine Verletzung der Treuepflicht dar, wenn es sich im Rahmen der Sitte

\footnotetext{
${ }^{9}$ OLG Wien 17. 9. 1975, 6 R 112/75.

${ }^{10} \mathrm{OGH}$ 27. 2. 1985,4 Ob 520/84.

${ }^{11} \mathrm{OGH}$ 18. 3. 1965, 5 Ob 273/64.
}

und des Anstandes hält. Selbst das Eingehen von Situationen, welche die geschlechtliche Erregung oder Bestätigung begünstigen oder gewöhnlich zum Ziel haben, wie z.B. das Übernachten in einem Zimmer, ist nicht als Eheverfehlung anzusehen, wenn diesem Verhalten kein erotischer Beweggrund sondern eine durch die Umstände geschaffene Zwangslage zugrunde liegt und es tatsächlich nicht zu Äußerungen erotische Regungen kommt.

Es kann jedoch auch ein solcher gegen den ausdrücklichen oder erkennbar gezeigten Willen des anderen Gatten fortgesetzter Verkehr eine schwere Eheverfehlung bedeuten, weil er unter gewöhnlichen Verhältnissen eine Entfremdung zwischen den Ehegatten hervorzurufen, jedenfalls aber zu vertiefen geeignet war. ${ }^{\text {} 12}$

Sinngemäß deckungsgleich z.B.:

„Die eheliche Treuepflicht ist nicht auf den sexuellen Bereich beschränkt. Auch rein „freundschaftliche" Beziehungen können Eheverfehlungen darstellen, wenn sie gegen den Willen des anderen Ehegatten gepflogen werden oder wenn ein Ehegatte sie dem anderen trotz ihrer über das Übliche hinausgehenden Intensität verheimlicht. " ${ }^{13}$

Wiederholt hatte sich der OGH ab den 70erJahren auch mit Frage der Sexualfreiheit zu beschäftigen:

„Die zwischen Ehegatten getroffene Vereinbarung der Sexualfreiheit verstößt gegen das Wesen der Ehe und vermag als sittenwidriger Vertrag keine rechtsverbindlichen Wirkungen hervorzubringen. Das bedeutet, dass der Ehebruch seinen objektiven ehewidrigen und sittenwidrigen Charakter und damit seine Eigenschaft als Scheidungsgrund nach § 47 EheG nicht verliert. Es gibt kein Recht auf Ehebruch, das aus einer derartigen Zustimmung des anderen Ehegatten abgeleitet werden kann. Die Zustimmung des

\footnotetext{
12 OGH 18. 3. 1965, 5 Ob 273/64.

${ }^{13}$ LG Salzburg 30. 4. 2008, 21 R 552/07h.
} 
anderen Ehegatten bedeutet für die Dauer ihres aufrechten Bestandes (bei jederzeitiger Widerruflichkeit) nur ein Hindernis für die Geltendmachung des Ehebruches iSd $\S 47$ Abs. 2 EheG. Auf den guten Glauben, des Ehebrechers sein Verhalten werde vom Ehepartner im Sinne einer Zustimmung geduldet, kommt es nicht an. ${ }^{\prime 14}$

Knapp vor der Abschaffung des absoluten Scheidungsgrundes hatten sich die Gerichte auch noch mit dem Thema Gruppensex zu beschäftigen:

„Kam es zu den sexuellen Beziehungen zwischen der Klägerin und Herrn M erst, nachdem im Zuge eines Gespräches zwischen den Männern der Klägerin der Vorschlag gemacht wurde, Gruppensex zu betreiben, so liegt Zustimmung zum Ehebruch vor. ${ }^{\text {}}{ }^{15}$

Heute haben die Internetforen in der Judikatur ihren Niederschlag gefunden:

„Dass der Kläger per Internet auf einer als ,Flirtstar' bezeichneten Website Kontakt suchte und das Interesse zur Kontaktaufnahme dadurch anzuregen versuchte, dass er im Internet ein Foto seiner Person nur mit Unterhose bekleidet veröffentlichte, stellt eine Verletzung der ehelichen Treue dar. ${ }^{\prime 16}$

\section{Verweigerung der Fortpflanzung}

Gleich nach dem Krieg wurde ein für allemal klargestellt:

„Eine während der Ehe eingetretene Impotenz begründet aber ohne Nachweise eines Verschuldens keinen Scheidungsgrund nach $\S 48$ EheG. ${ }^{117}$

Der Scheidungsgrund des $\S 48$ EheG ist im Gegensatz zu der in der Zeit des Nationalsozialismus vertretenen Meinung schon dann zu ver-

\footnotetext{
${ }^{14}$ OGH 24. 4. 1975, 6 Ob 51/75.

${ }^{15}$ LGZ Wien 9. 5. 1995, 45 R 2062/95.

${ }^{16}$ LGZ Wien 7. 3. 2007, 43 R 89/07f.

${ }^{17}$ OLG Wien 14. 3. 1946, 1 R 119/46.
}

neinen, wenn der Ehe auch nur ein gesundes Kind entsprossen ist. Die Weigerung weitere Kinder zu empfangen, kann allerdings einen Scheidungsgrund nach $\S 49$ EheG bilden. ${ }^{18}$

Überhaupt waren Scheidungen nach § 48 EheG eher die Ausnahme:

„In der von der Beklagten am Beginn der Ehe einmal geäußerten Meinung, es sei im Hinblick auf die wirtschaftlichen Verhältnisse wohl besser, in den ersten beiden Ehejahren noch keine Kinder zu haben, kann noch keineswegs eine beharrliche Verweigerung zur Empfängnis von Nachkommenschaft im Sinne des §48 EheG erblickt werden. ${ }^{19}$

Wenn sich eine berufstätige Ehefrau nach 10jähriger Ehe im Hinblick auf in Aussicht stehende Doppelbelastungen weigert, Nachkommenschaft zu bekommen, so liegt darin keine beharrliche Weigerung“ i.S.d. § 48 EheG. „Diese Bestimmung ist im Zusammenhang mit den sonstigen Lebensverhältnissen $\mathrm{zu}$ sehen und kann nicht zur Überforderung eines Partners führen. “20

Interessant eine Entscheidung zum aktuellen Eherecht:

„Die Verweigerung der Fortpflanzung stellt auch nach Aufhebung des $\S 48$ EheG einen Scheidungsgrund dar. Gemäß $§ 44$ ABGB gehört die Fortpflanzung zum Wesen der Ehe. Kein Ehepartner ist berechtigt, die Empfängnis gegen den Willen des anderen zu verhindern. Eine Vereinbarung zwischen den Ehegatten dass die Ehe kinderlos bleiben soll, ist jedoch zulässig. Nur bei Fehlen einer solchen Vereinbarung ist die beharrliche und unbegründete Fortpflanzungsverweigerung ein Scheidungsgrund iSd § 49 EheG. “21

\footnotetext{
${ }^{18}$ OGH 7. 7. 1966, 6 Ob 202/66.

${ }^{19}$ OGH 20. 5. 1965, 5 Ob 100/65.

${ }^{20}$ LGZ Wien 20. 6. 1995, 43 R 2046/95.

${ }^{21}$ LGZ Wien 26. 11. 2009, 45 R 507/09f.
} 
Von der Verweigerung der Fortpflanzung klar $\mathrm{zu}$ trennen ist die Verpflichtung zum ehelichen Geschlechtsverkehr.

\section{Verweigerung des Geschlechts- verkehrs}

Unbestritten ist, dass die grundlose Verweigerung des Geschlechtsverkehrs seit jeher bis heute eine schwere Eheverfehlung darstellt. Auch hierzu gibt es eine Vielzahl an Entscheidungen:

„Da die Verbindlichkeit zur Leistung der ehelichen Pflichten die ursprünglichste, natürlichste und damit auch die wichtigste Funktion der Ehe ist, ist die Verweigerung des Geschlechtsverkehrs grundsätzlich eine schwere Eheverfehlung es sei denn, dass triftige Gründe dafür vorliegen. Solche Gründe können physischer (Erkrankungen, bei denen der Geschlechtsverkehr Schmerzen auslöst oder die Heilung verzögert) oder psychischer Natur (Kränkungen jeglicher Art, die das Ausmaß von Scheidungsgründen angenommen haben) sein. In solchen Fällen stellt der Verweigerung der Geschlechtsverkehr keine Eheverfehlung dar. “22

„Eine hauptsächlich aus der Verschiedenheit der Veranlagung aber auch aus religiösen Motivierungen sich ergebende Unstimmigkeit hinsichtlich der sexuellen Bedürfnisse vermag noch nicht dafür auszureichen, dem Ehegatten der die sexuellen Wünsche des anderen nicht restlos zu befriedigen vermag, eine schwere Eheverfehlung anzulasten, sofern nicht überhaupt eine Verweigerung der ehelichen Pflichten festzustellen ist. " 23

„Sache des Ehemannes wäre es gewesen, sich der Ehefrau, nachdem sie sich von einer schwe-

\footnotetext{
${ }^{22} \mathrm{OGH}$ 2. 12. $1970,5 \mathrm{Ob} 241 / 70$.

${ }^{23} \mathrm{OGH}$ 9. 12. 1975, $5 \mathrm{Ob} 236 / 75$.
}

ren Bauch- und Unterleibsoperation erholt hatte, in sexueller Hinsicht wieder zu nähern. ${ }^{{ }^{2} 24}$

2011 würde das Entscheidungsorgan wohl fragen, warum die Ehefrau nichts gesagt hat ...

„Die Verweigerung des Geschlechtsverkehrs durch die Ehefrau ist dann keine Eheverfehlung, wenn der Mann betrunken nach Hause kommt und Andeutungen über andere Frauen macht. “25 „Die Verweigerung des Geschlechtsverkehrs nach unmittelbar vorangegangenen erfolglosen Gesprächen über die beabsichtigte Scheidung und die finanziellen Auseinandersetzungen war berechtigt. ${ }{ }^{26}$

\section{Beschimpfungen, Verletzung der Pflicht zur anständigen Begeg- nung}

Der "Dauerbrenner" in Scheidungsverfahren sind auch seit jeher Beschimpfungen als Ausfluss der Verletzung der Pflicht zur anständigen Begegnung. Der Begriff der Zanksucht (wie der Titel des Vortrages lautet) ist in keiner einzigen Entscheidung enthalten, jedoch der Begriff der Widerspruchssucht der Ehefrau:

„Andauerndes liebloses und geradezu feindseliges Verhalten ständig als Närrin bezeichnet und dass sie aus einer Narrenbrut kommt ist schwere Eheverfehlung. ${ }^{27}$

„Wer durch sein unbeherrschtes Wesen dem anderen Ehegatten Angst, Sorge und Aufregung bereitet, wer ständig an der voll des guten Willens geleisteten Arbeit des anderen Teiles ungerecht und undankbar nörgelt, begeht eine schwere Eheverfehlung. ${ }^{\prime 28}$

\footnotetext{
${ }^{24}$ OGH 20. 11. 1975, 7 Ob 244/75.

${ }^{25}$ OLG Wien 16. 12. 1964, 6 R 120/64.

${ }^{26}$ OGH 20. 5. 1981, 1 Ob 609/81.

27 OLG Wien 9. 4. 1946.

${ }^{28}$ OGH 26. 5. 1966, 5 Ob 41/66.
} 
„Ständige mit Beschimpfungen verbundenen Streitigkeiten und Rechthabereien sind vor allem dann als schwere Eheverfehlungen anzusehen, wenn sie über einen sehr langen Zeitraum immer wieder herbeigeführt werden. ${ }^{29}$

„Der Gebrauch des Schimpfwortes ,Verbrecher" durch die Beklagte, als sie von den ehewidrigen Beziehungen des Klägers erfuhr, ist zwar nicht zu billigen, aber doch eine sehr verständliche Reaktionshandlung und damit keine schwere Eheverfehlung." ${ }^{30}$

„Mag auch der Ausdruck ,Dreckblunze' mit Rücksicht auf das Milieu der Streitteile nicht ins Gewicht fallen, so ist doch auch in den Kreisen, denen die Ehegatten angehören, eine Beschuldigung, die Frau habe eine Goschn wie eine Schleiferin` eine schwere Beleidigung. “31

„Die Beschimpfungen mit dem Wort ,Feigling” sind auch unter Berücksichtigung des Standes der Streitteile (Wirtschaftstreuhänder) keineswegs schwerwiegend. “ ${ }^{32}$

Aber nicht nur Streit sondern auch Kommunikationsabbruch stellt u.U. eine schwere Eheverfehlung dar:

Tagelanges beharrliches Schweigen eines Ehegatten kann, wenn es sich häufig wiederholt, eine schwere Eheverfehlung bilden. ${ }^{33}$

Gesprächsverweigerung durch zwei Wochen ist schwere Eheverfehlung. ${ }^{34}$

\section{Gewalt in der Ehe}

In keinem anderen Bereich des Scheidungsrechtes hat in den letzten 60 Jahren eine solche Kehrtwendung stattgefunden wie im Bereich Gewalt in der Ehe, so kann man in älteren Ent-

${ }^{29}$ OGH 7. 7. 1981, 5 Ob 662/80.

${ }^{30}$ OLG Wien 28. 5. 1965, 6 R 130/65.

${ }^{31}$ OGH 10. 4. 1954, 2 Ob 942/53.

${ }^{32}$ OLG Wien 19. 3. 1965, 5 R 41/65.

${ }^{33}$ OGH 20. 11. 1975, 7 Ob 244/75.

${ }^{34}$ OGH 29. 3. 1990, 6 Ob 503/90. scheidungen nachlesen, dass kleinere Tätlichkeiten gar nicht als Eheverfehlungen $\mathrm{zu}$ werten sind und Begriffe wie "sanfte Gewalt" sind heute nicht mehr in Urteilen zu finden ...

„Als spontane nicht als Eheverfehlung zu wertende Reaktionshandlung ist auch eine Ohrfeige $\mathrm{zu}$ beurteilen, welche die Beklagte dem Kläger wegen der vorausgegangen Beleidigung ihrer Eltern versetzt hat. ${ }^{\prime 35}$

„Eine tätliche Beleidigung ohne jede Folge, die vom anderen Ehegatten provoziert wurde, stellt keine schwere Eheverfehlung dar. “ ${ }^{36}$

„Milieubedingte Entgleisungen darf nicht das Gewicht einer schweren Eheverfehlung beigemessen werden. “37

„Der Ehemann darf keine Gewalt gegen seine Gattin zur Erzwingung des Geschlechtsverkehrs anwenden. Es blieb aber offen, ob der Beklagte bei dem Versuch den ehelichen Verkehr zu erzwingen mit brutaler Gewalt oder ob er etwa sanfte Gewalt angewendet hat. “38

„Nicht nur Beschimpfungen sondern selbst Misshandlungen mit leichten Verletzungsfolgen können unter Umständen bei Gegenüberstellung mit dem Verhalten des anderen Ehegatten begreifliche Reaktion sein und somit keine schwere Eheverfehlung. " ${ }^{39}$

In diesem Zusammenhang soll auf die Entscheidung des LGZ Wien verwiesen werden, die besagt:

„Die Verabreichung von Ohrfeigen und die Bedrohung des Ehepartners durch Ansetzen einer Klinge eines $30 \mathrm{~cm}$ langen Messers an den Hals mit dem Beifügen, die Bedrohte umzubringen (noch dazu, wenn die Klägerin das gemeinsame Kind in den Armen hält und dadurch besonders wehrlos ist), stellt keine entschuldbare Reakti-

\footnotetext{
${ }^{35}$ OLG 10. 2. 1965, 6 R 9/65.

${ }^{36}$ OGH 5. 6. 1964, 5 Ob 110/64.

${ }^{37}$ OGH 16. 6. 1964, 8 Ob 79/64.

${ }^{38}$ OLG Wien 11. 1. 1965, 8 R 291/64.

${ }^{39}$ OLG Wien 27. 4. 1981, 14 R 59/81.
} 
onshandlung auf die Ankündigung sich scheiden zu lassen dar. ${ }^{40}$

Dies bedeutet aber, dass noch 1998 bei Ohrfeigen und Morddrohung mit einem Messer versucht wurde, dies als entschuldbare Reaktionshandlung darzustellen!

Im Jahr 2000 hielt das LG Salzburg fest: „Tätlichkeiten können nie als entschuldbare Reaktion auf ein Fehlverhalten des anderen gewertet werden. ${ }^{41}$

In der neueren Judikatur wird auch die Bedrohung mit Gewalt als schwere Eheverfehlung gesehen:

„Der Beklagte hat gegen die Klägerin im Streit die Hand drohend erhoben als ob er sie schlagen wollte und mit den Füßen gegen den Mistkübel gestoßen, sodass dieser umfiel - Eheverfehlung. ${ }^{442}$

\section{Haushaltsführung}

Bei einer Meinungsumfrage, was alles unter dem Begriff der Eheverfehlung zu verstehen ist, würde die Vernachlässigung der Haushaltspflichten wohl viele Punkte bekommen, viele Menschen wissen, dass der Haushalt gut geführt werden muss. Tatsächlich sind Scheidungen aus diesem Grund die regelrechte Ausnahme:

„Die Vernachlässigung des Haushaltes ist im allgemeinen nur dann eine schwere Eheverfehlung, wenn es sich um eine arge oder über längere Zeit anhaltende und auf Böswilligkeit beruhende Vernachlässigung handelt. “43

„Unpünktlichkeit bei der Essenszubereitung mag im Allgemeinen keine schwere Eheverfehlung sein. Dies kann aber dann nicht gelten, wenn derartige Nachlässigkeiten ständig oder doch häufig vorkommen, vor allem aber beson-

\footnotetext{
${ }^{40}$ LGZ Wien 13. 7. 1998, 44 R 537/98s.

4121 R 542/99.

${ }^{42}$ LGZ Wien, 13. 5. 2009, 43 R 263/09.

${ }^{43}$ OGH 9. 9. 1965, 5 Ob 82/65.
}

dere Gründe, wie z.B. der Gesundheitszustand des anderen Ehegatten oder beruflichen Notwendigkeit die Pünktlichkeit der Mahlzeiten erforderlich machen. “44

„Dauernde und grobe Vernachlässigung der Hausfrauenpflichten durch die nicht berufstätige Ehefrau bildet eine schwere Eheverfehlung. “45

„Bei Prüfung der Frage ob im Einzelfall die Art der Haushaltsführung bzw. die Vernachlässigung der Betreuung des Haushaltes als schwere Eheverfehlung zu qualifizieren ist, ist zu untersuchen, welche Maßstäbe an die Haushaltführung anzulegen sind, wobei die persönliche Ansicht beider Ehegatten zu dieser Frage wesentlich ist, zumal die Anforderungen an die Ordnung nicht bei allen Menschen in gleicher Weise ausgeprägt sind. Ergibt sich, dass die Art der Haushaltsführung diesen Voraussetzungen nicht entspricht, so ist bei gravierenden Abweichung zu untersuchen, auf welche Gründe eine allfällige Vernachlässigung zurückzuführen und ob diese vorwerfbar ist. ${ }^{4} 46$

Interessant erscheint in diesem Zusammenhang auch der Fall eines Ehepaares; sie Österreicherin und er Türke. Hier stellte sich u.a. die Frage, ob man beim Kochen auf die kulturellen Vorlieben des Partners eingehen muss. Sie kochte zwar kein Schweinefleisch, kümmerte sich aber überhaupt nicht um den türkischen Geschmack. Das Erstgericht verneinte jegliche Relevanz, da eine gewisse Toleranz des Klägers für die Gepflogenheiten des Gastlandes vorauszusetzen sei. Das Berufungsgericht fand, dass das mangelnde Bemühen der Ehefrau um türkische Rezepte sei zwar für sich selbst nicht als schwere Eheverfehlung zu werten, passe aber in das sich bietende Bild, dass die Beklagte nicht bereit gewesen sei, ihre Interessen und Lebensvorstellungen mit

\footnotetext{
${ }^{44}$ OLG Wien 19. 3. 1965, 5 R 41/65.

${ }^{45}$ OLG Wien 11. 12. 1975, 7 Ob 259/75.

${ }^{46}$ OLG Wien, 7. 5. 1985, 12 R 105/85.
} 
denen des Ehepartner auszugleichen und einvernehmlich $\mathrm{zu}$ gestalten. Letztlich blieb die Frage des Kochens unbeantwortet; der OGH sprach aus, dass das Verbringen der spärlichen Freizeit ohne Partner eine schwere Eheverfehlung ist, denn Hauptthema des Verfahrens war, dass er seine türkischen Freunde getroffen hat und sie mit ihren Freundinnen Werbefahrten unternommen hat. ${ }^{47}$

\section{Unterhalt}

Viel häufiger als um Haushaltspflichten geht es bei der Scheidung um Geld:

„Die Vernachlässigung der Unterhaltspflicht während aufrechter Ehe stellt eine schwere Eheverfehlung dar, die durch längere Zeit fortgesetzt, die Zerrüttung der Ehe herbeizuführen geeignet ist." ${ }^{48}$

„Der Beklagten ist anzulasten, dass sie durch mehrere Monate hindurch vom Konto des Klägers Geldbeträge abhob, ohne ihm auf sein Verlangen Rechenschaft über die Verwendung des Geldes zu geben. Hierzu war die Beklagte umso mehr verpflichtet als ihr der Kläger für größere Wochenendeinkäufe aber auch für sonstige kleiner Einkäufe weiters für die Anschaffung von Bekleidung, gesonderte Geldbeträge zur Verfügung gestellt hat. Die Äußerung der Beklagten, sie habe die Beträge „eben gebraucht" war unzureichend. Die Verweigerung jeder Rechenschaft stellt eine schwere Eheverfehlung i.S.d. $\S 49$ EheG dar." 49

Wenn es darum geht, ausreichend Geld für das gemeinsame Leben zur Verfügung zu haben, ist die Frage der Berufstätigkeit und der Vereinbarung von Beruf und Familienleben ein immer wichtiger werdender Themenkomplex in Scheidungsverfahren.

\footnotetext{
${ }^{47}$ OGH 28. 2. 1990, 2 Ob 521/90.

${ }^{48}$ OLG Wien 14. 9. 1965, 7 R 169/65.

${ }^{49}$ OGH 28. 8. 1985, 1 Ob 631/85.
}

Noch 1966 entschied der OGH:

„Der Antritt einer Stellung ist der Klägerin- ungeachtet der Besorgnisse um ihren Unterhalt als Eheverfehlung anzulasten, weil sie mit dem Beklagten vorher keine Rücksprache genommen hat. “50

Dem gegenüber zwei Entscheidungen aus 1975

„Eine Berufstätigkeit der Ehefrau ohne Kenntnis des Ehemannes ist nur dann ein ehewidriges Verhalten, wenn der Ehemann im Falle der Kenntnis dieser Berufstätigkeit ernstlich und aus gerechtfertigten Gründen widersprochen hätte. ${ }^{\prime 51}$

„Die Weigerung in der Landwirtschaft des Ehemannes mitzuarbeiten, ist eine schwere Eheverfehlung. ${ }^{\circ 2}$

Über die Arbeitsmoral am Bauernhof im Jahr 1954:

„Das Verhalten der Ehefrau, die nicht nur spät aufstand, die ihr zugewiesene Arbeit mangelhaft und unwillig versah und die Arbeit der Schwiegermutter kritisierte, was ihr überhaupt nicht zustand, sondern auch mehrmals den Hof bloß aus dem Grund verließ, um sich landwirtschaftlichen Arbeiten zu entziehen, die ihr nicht genehm waren, widersprach gröblichst der auf einem Bauernhof geltenden Arbeitsmoral und muss als die eigentliche Ursache für die bald eingetretene Zerrüttung der Ehe angesehen werden. Es wäre auch die Pflicht des Ehemannes gewesen, die Gegensätze zwischen ihr und seinen Eltern auszugleichen. Zweifellos ist ihm in dieser Richtung eine schwere Eheverfehlung anzulasten, sie kann aber nicht als besonders gravierend angesehen werden, wenn er als der arbeitende Sohn gegenüber den besitzenden Eltern und besonders der energischen Mutter nicht aufkommen konnte. Dem Ehemann kann auch nicht zum überwiegenden Verschulden

\footnotetext{
${ }^{50}$ OGH 1. 3. 1966, 8 Ob 48/66.

${ }^{51}$ OLG Wien 21. 10. 1975.

52 OLG Wien 12. 2. 1975, 6 R 5/75.
} 
angerechnet werden, dass es nach öfteren Gasthausbesuchen einmal zu Schlägen gegen seine Gattin und zu deren Beschimpfung mit „Drecksau" gekommen ist. All diese Vorwürfe können auf die schwere Eheverfehlung bildende Arbeitsunwilligkeit der Ehefrau und die dadurch entstandenen Spannungen zurückgeführt werden, denen der Ehemann teils zu entgehen teils Reaktionshandlungen entgegensetzte. " 53

Ein anderer Bauernhof im Jahr 1985:

„Hatte die Klägerin eine erhöhte Mitarbeit durch einvernehmliche gemeinsame Übernahme der schwer verschuldeten Landwirtschaft in Kauf genommen, konnte sie nicht ohne Einvernehmen mit dem Beklagten sich auf den Standpunkt stellen als Hälfteigentümerin nur mehr die Hälfte des vorhandenen Viehs versorgen zu müssen, so dass die andere Hälfte sowie die Schweinezucht und die Hühnerhaltung dem Beklagten oblagen. Insbesondere die Hühnerhaltung gehört überdies auch üblicherweise zu den Aufgaben einer Bäuerin. Die Vorinstanzen haben daher zu Recht der Klägerin ihre Weigerung im bisherigen Ausmaß in der Landwirtschaft mitzuarbeiten als Eheverfehlung angelastet.

In der partnerschaftlichen Ehe ist es Pflicht der Ehegatten widerstreitende berufliche Vorstellungen zu koordinieren, insbesondere ist bei der Wahl und Ausübung der Erwerbstätigkeit auf die Belange des anderen Ehegatten und der Familie die gebotene Rücksicht zu nehmen. Jeder Ehegatte ist verpflichtet, seine Berufsarbeit einzuteilen und sich in seiner von berechtigten Erwerbsstreben und beruflichen Ehrgeiz getragenen Berufsausübung so einzuschränken, dass auch die Belange des anderen Ehegatten und der Familie gewahrt bleiben. ${ }^{\text {"54 }}$

Diese Entscheidung aus dem Jahr 1985 erscheint richtungsweisend für ein Voranstellen des Familienlebens vor dem Berufsleben! Allerdings

\footnotetext{
${ }^{53} \mathrm{OGH}$ 1. 12. 1954, $1 \mathrm{Ob} 893 / 54$.

${ }^{54}$ OGH 16. 4. 1985, 2 Ob 534/85.
}

kommt es auch hier wieder auf die Umstände des Einzelfalls an:

„Es ist allerdings allgemein bekannt, dass die berufliche Belastung eines Wirtschaftsanwalts weit über dem Durchschnitt liegt, was der Beklagten aufgrund jahrelanger vorehelicher Lebensgemeinschaft bei Eingehen der Ehe auch bewusst war. Eine schwere Eheverfehlung des Klägers kann daher nur darin erblickt werden, dass er zusätzlich zu seiner starken beruflichen Inanspruchnahme auch noch häufiger private Termine abends wahrnahm und deswegen $\mathrm{zu}$ wenig Zeit für das Eheleben aufwendete.“55

\section{Eheverfehlung in Bezug auf das Familienleben mit Kindern}

Zunächst eine Entscheidung aus dem Jahr 1965, die heute wirklich antiquiert aussieht, denn heute wäre die Scheidung unter diesen Voraussetzungen wohl schon längst vorher vollzogen worden:

„Die Unterlassung der Mitteilung über die Geburt des Kindes ist keine schwere Eheverfehlung, wenn der Beklagte jegliches Interesse an dem zu erwartenden Kind fehlen ließ, für die Klägerin während der Schwangerschaft trotz ihres schlechten Gesundheitszustandes nie ein gutes Wort gefunden hat, sich äußerte weder auf sie noch auf das Kind Rücksicht zu nehmen de Klägerin den Zeitpunkt der voraussichtlichen Geburt nicht hat vorhersehen können, die Geburt schwer war, das Kind sogleich nach der Geburt getauft wurde und die Klägerin sich nicht neuen Aufregungen hat aussetzen wollen. ${ }^{\prime 56}$

Probleme in der Zweit- und Drittehe mit den Kindern aus einer vorangehenden Beziehung sind kein neues Problem der Patchwork-Familiy

\footnotetext{
${ }^{55}$ LGZ Wien 12. 5. 2009, 44 R 194/09r.

${ }^{56}$ OLG Wien 19. 2. 1965, 5 R 11/65.
} 
und kommen in ganz unterschiedlichen Ausprägungen in der Judikatur vor:

„Die mangelnde Bereitschaft mit den Kindern des anderen Ehegatten aus erster Ehe, mit denen dieser ein gutes Einvernehmen unterhält, gut auszukommen, wodurch die Zerrüttung der Ehe mit eingeleitet wurde, stellt eine schwere Eheverfehlung dar. ${ }^{\prime \prime}{ }^{7}$

„Das Aufhetzen der Kinder gegen den eigenen Vater stellt eine schwere Eheverfehlung dar, weil es nicht nur eine Verleitung der Kinder zur Verletzung der ihnen vom Gesetz auferlegten Pflicht, den Eltern Ehrfurcht und Gehorsam zu erweisen (§ 144 ABGB) sondern auch einen Verstoß gegen die natürliche Sittenordnung darstellt." ${ }^{\text {" }} 8$

„Schwere Eheverfehlung - ohne Wissen und hinter dem Rücken des Partners Durchführung von Zuwendungen aus den von den Streitteilen gemeinsam geschaffenen Vermögen an den geldfordernden Sohn. ${ }^{\text {"59 }}$

„Die Behinderung und Verweigerung des Besuchsrechtes des Klägers durch die Beklagte stellt - als Verstoß gegen die Pflicht zur Respektierung der beiderseitigen Rechte der Kinder gegenüber - eine schwere Eheverfehlung dar." ${ }^{\prime 60}$ Überhaupt spielen die Besuchsrecht gestern wie heute eine entscheidende Rolle:

„Nur wenn die Klägerin grundlos den persönlichen Kontakt zwischen dem Kind und seinem Vater aufheben oder in ungebührlicher Weise beschränken und so ihm das Kind entfremden wollte, wäre ihr die Weigerung, den Aufenthaltsort des Kindes bekanntzugeben und das Kind an den Beklagten auszufolgen, als Verfehlung anzulasten. Sollte sich die Klägerin in begründeter Sorge um das Wohl des Kindes so verhalten haben, dann könnte in diesem Verhal-

\footnotetext{
${ }^{57}$ OGH 17. 2. 1965,6 Ob 349/64.

${ }^{58}$ OGH 14. 7. 1954, 8 Ob 217/54.

${ }^{59}$ LGZ Wien 9. 2. 1996, 45 R 1195/95h.

${ }^{60}$ OGH 26. 3. 19855 Ob 557/84.
}

ten eine Verfehlung der Klägerin nicht erblickt werden. ${ }^{\text {}}{ }^{1}$

Grundsätzlich hat das LGZ Wien zu diesem Themenkomplex folgerichtig festgestellt: „Hineinziehen der Kinder in einen Ehestreit ist eine schwere Eheverfehlung." ${ }^{62}$

Auch die Schwiegereltern sind Thema mancher Entscheidungen geworden:

„Unbegründetes Verlangen, der Ehepartner solle Besuche bei seinen Eltern unterlassen, ist eine schwere Eheverfehlung." ${ }^{63}$

Umgekehrt:

„Es ist als ehewidriges Verhalten anzulasten, wenn der Mann der dominanten Einflussnahme seiner Mutter auf familieninterne Angelegenheiten nicht entgegentritt. ${ }^{\prime \prime}{ }^{6}$

\section{Liebloses Verhalten}

Zuletzt zeigt sich in der Judikatur die Tendenz viele Handlungen, die letztlich einen Ehepartner dazu bewegen die Scheidungsklage einzureichen, unter dem umfassenden Begriff des lieblosen Verhaltens zu subsumieren, dazu gehört auch der „Klassiker" des „ständig-spätNachhausekommens". Dies zeigt aber auch, welch verschiedene Vorwürfe zur Rechtfertigung einer Scheidungsklage herangezogen werden, was den Wunsch der Richterschaft nach einer Abschaffung des Verschuldensprinzip nachvollziehbar macht.

In der Folge sind einige Beispiele für liebloses Verhalten aufgezählt

"Je geringer die Freizeit des Klägers gewesen ist, desto schwerer wiegt seine Verfehlung seine Freizeit nicht mit seiner Frau und seinen 3 Kindern sondern beim Kartenspielen mit seinen

\footnotetext{
${ }^{61}$ OLG Wien 19. 2. 1965, 5 R 11/65.

${ }^{62}$ LGZ Wien 10. 3. 1999.

${ }^{63}$ OGH 7. 3. 1990, 1 Ob 518/90.

${ }^{64}$ LGZ Wien 14. 10. 1996, 44 R 643/96a.
} 
Eltern und bei einem Chor, bei Veranstaltungen dieses Chores, bei Kegelabenden und Feiern mit den Kameraden verbracht zu haben. ${ }^{\text {"65 }}$

„Die Weigerung der Ehefrau, dem Ehemann, die von seinem Geld gekauften Säuglingssachen zu zeigen, und die Bestimmung des Namens des ehelichen Kindes ohne Rücksprache mit dem Ehemann sind schwere Eheverfehlungen." 66

„Der Beklagten muss vorgeworfen werden, dass sie auch gegenüber Patienten die beruflichen Fähigkeiten des Klägers immer wieder herabgesetzt und dem gegenüber ihre eigenen angeblich größeren Fähigkeiten hervorgehoben hat (beide Streitteile sind Dentisten). Ein derartiges Verhalten muss als ein wesentlicher Angriff auf die Selbstachtung eines im Berufsleben stehenden Mannes gewertet werden, und stellt daher schon für sich eine schwere Eheverfehlung dar. ${ }^{\text {"67 }}$

„Der Umstand, dass der Kläger ein starker Raucher ist, was die Beklagte seit jeher wusste, stellt sicher keine schwere Eheverfehlung dar. Berücksichtigt man aber, dass der Kläger auch abends im einzigen Zimmer der Ehewohnung viel rauchte und untersagte, dass die Fenster des Zimmers geöffnet werden, ergibt sich, dass er sich gegenüber der Beklagten, die dies als störend empfand, rücksichtslos und ehewidrig verhält. “68

„Wenn auch eine öffentliche Stellungnahme zu politischen Fragen in einer dem Partner nicht genehmen Art mit dem Inhalt, die seinen politischen Ansichten widerspricht, keine Eheverfehlung darstellt, ist doch das Vertreten solcher Ansichten innerhalb der Familie mit missionarischen Eifer, d.h. in einer Weise, die dem Ehepartner und den Kindern die eigene Meinung intolerant aufzwingen will, eine Eheverfehlung. ${ }^{.69}$

\footnotetext{
${ }^{65}$ OLG Wien 7. 10. 1965, 8 R 177/65.

${ }^{66}$ OLG Wien 12. 2. 1975.

${ }^{67}$ OGH 12. 6. 1980, 7 Ob 604/80.

${ }^{68}$ OGH 20. 5. 1981, 6 Ob 609/81.

${ }^{69}$ OGH 29. 4. 1981, 1 Ob 540/81.
}

Planen der Sommerferien durch den Beklagten ohne Rückfrage bei der Klägerin - schwere Eheverfehlung. ${ }^{70}$

Aber auch schon unerwünschte Telefonanrufe an der Arbeitsstelle des Ehepartners können eine schwere Eheverfehlung sein. ${ }^{71}$

„Eine Eheverfehlung ist das Verheimlichen von für beide Teile wichtigen Umständen, wie etwa das verschleiern einer kritischen Wirtschaftslage des eigenen Unternehmens. Wenn daher der Beklagte der offensichtlich für die Kredite seines Unternehmens persönlich haftet, hohe Schulden für seine Firma eingeht, wäre er aus dem Treuverhältnis seiner Gattin gegenüber verpflichtet, diese entsprechend $\mathrm{zu}$ informieren und die finanzielle Situation seines Betriebs vorab zu besprechen. ${ }^{\text {"72 }}$

Verbringen der Freizeit immer mehr ohne Partner (hier: Wandern, ohne der hilfsbedürftigen Gattin den Aufenthaltsort bekanntzugeben schwere Eheverfehlung. ${ }^{73}$ )

Fortgesetztes Betrachten von Aktfotos nackter Frauen ungeachtet der Gegenwart der Ehefrau schwere Eheverfehlung. ${ }^{74}$

Wer jetzt den Eindruck bekommen hat, dass fast alles Eheverfehlung sein kann: JA das stimmt. Es kommt immer auf den Zusammenhang an oder wie das LGZ formulierte:

"Gerade bei der Beurteilung der Zerrüttungskausalität einer Eheverfehlung und des Ursachenzusammenhangs muss die gesamte Geschichte der Ehe, deren Verlauf und die Entstehung sowie die Entwicklung der Konflikte betrachtet werden. “75

Folgende Sachverhalte wurden z.B: nicht als Eheverfehlung angesehen:

\footnotetext{
${ }^{70}$ LGZ Wien 26. 3. 1996, 43 R 162/96h.

${ }^{71}$ LGZ Wien 24. 12. 1999, 43 R 830/99y.

${ }^{72}$ LGZ Wien 22. 10. 2008, 48 R 280/08v.

${ }^{73}$ LGZ Wien 11. 2. 2002, 42 R 550/01w.

${ }^{74}$ LGZ 13. 5. 2009, 43 R 263/09h.

${ }^{75}$ LGZ Wien 29. 5. 2007, 45 R 754/06z.
} 
„Abdrehen des Radios, das von der Ehefrau nur für den Hund aufgedreht wird, durch den Ehemann, der eine Musikberieselung nicht will, ist keine schwere Eheverfehlung. ${ }^{476}$

„Die Klägerin hatte die Vermutung häufiger Anrufe zu einer anderen Frau. Wenn sie, um diese ausfindig zu machen, gegen Datenschutzbestimmungen verstößt, ist ihr dieses Verhalten nicht vorwerfbar. ${ }^{" 77}$

„Soweit die Beklagte - entgegen wiederholten Ankündigungen und der Kritik des Klägers zum Trotz - das Rauchen nicht eingestellt hat, kann dem Scheitern eines Ehegatten beim Versuch das Rauchen aufzugeben, objektiv nicht die für einen Scheidungsgrund gemäß § 49 EheG erforderliche Zerrüttungswirkung beigemessen werden. ${ }^{\prime \prime}{ }^{78}$

Von zunehmender Bedeutung wird die Frage, ob schlechte Angewohnheiten zur Eheverfehlung werden können, damit muss im Scheidungsverfahren oft zunächst einmal geklärt werden, seit wann ein Partner Dinge tut oder Verhaltensweise an den Tag legt die möglicherweise ehewidrig sind:

„Ein Ehegatte darf vom anderen erwarten, dass er Neigungen, Gewohnheiten und Verhaltensweisen, die gedeihliches Zusammenleben stören soweit wie möglich unterdrückt. “79

„Es gibt weder ein Gewohnheitsrecht auf ehewidriges Verhalten noch als entsprechende Kehrseite den Verlust des Rechtes, ehewidriges Verhalten des Beklagten wegen lang dauernder Übung als Eheverfehlung geltend $\mathrm{zu}$ manchen. “80

Eine neuere Entscheidung geht hier in die andere Richtung:

\footnotetext{
${ }^{76}$ OLG Wien 19. 3. 1990, 14 R 12/90.

${ }^{77}$ LGZ Wien 18. 1. 2002, 42 R 546/01g.

${ }^{78}$ LGZ Wien 27. 1. 2009, 44 R 510/08p.

${ }^{79}$ LG Salzburg 18. 10. 2000, 21 R 168/00b.

${ }^{80}$ LGZ Wien 17. 7. 2000, 44 R 262/00f.
}

„Ein dem künftigen Ehegatten bekanntes Verhalten vor Eheschließung kann keine Eheverfehlung darstellen." ${ }^{\text {81 }}$

Es wird daher wie immer auf den konkreten Einzelfall ankommen ...

\section{Schlussbemerkung}

Wie anhand der vielen Beispiele aufgezeigt, erscheint die Beurteilung der Frage, was eine schwere Eheverfehlung ist, immer schwieriger. Der Scheidungsrichter wird zum Psychologen, wenn es darum geht die Entstehungsgeschichte eines Konflikts festzustellen. Ein Abgehen vom Verschuldensprinzip hin zum reinen Zerrüttungsprinzip wäre ein logischer Schritt, den schon viele europäische Länder gegangen sind.

Abschließend noch zwei sehr lebenspraktische Entscheidungen:

„Altersbedingte Veränderungen und insbes. eine Gewichtszunahme des Ehegatten im Verlauf einer jahrelangen Ehe in der auch Kinder geboren wurden, gehören zum gewöhnlichen Lebensrisiko. Es entspricht der Lebenserfahrung, dass eine erhebliche Gewichtszunahme über einen Zeitraum von 20 Jahren keineswegs ungewöhnlich ist. Nach den Feststellungen kann der Frau jedenfalls nicht angelastet werden, dass sie ihr Äußeres bewusst verändert hätte.“82

„Erhofften sich die Ehegatten eine mustergültige Ehe ohne Streit und Auseinandersetzung mit der völligen Befriedigung ihrer persönlichen Wünsche und scheitert diese Erwartung, so ist die Ehe dennoch nicht unheilbar zerrüttet, wenn angenommen werden kann, dass es ihnen gelingen wird, sich auf eine weniger idealistische Lebensform zu einigen und danach die Zukunft der Ehe zu gestalten." ${ }^{48}$

\footnotetext{
${ }^{81}$ LGZ Wien 7. 3. 2007, 43 R 89/07.

${ }^{82}$ LGZ Wien 27. 3. 1995, 44 R 2012/95.

${ }^{83}$ LG Salzburg 21. 1. 200923 R 643/08.
} 